\title{
Molecular Approach to the Nyctinastic Movement of the Plant Controlled by a Biological Clock
}

\author{
Minoru Ueda**, Noboru Takada and Shosuke Yamamura** \\ Laboratory of Natural Products, Department of Chemistry, Faculty of Science and Technology, Keio \\ University, Hiyoshi, Yokohama 223-8522, Japan \\ Tel.: (+81 (0) 45566 1702, Fax: +81 (0) 45566 1697, e-mail: ueda@chem.keio.ac.jp \\ **Author to whom correspondence should be addressed.
}

Received: 3 August 2001 / Accepted: 15 October 2001 / Published: 25 October 2001

\begin{abstract}
Most leguminous plants close their leaves in the evening, as if to sleep, and open them early in the morning. This circadian rhythm is known to be controlled by the biological clock of such plants. Extensive studies on other nyctinastic plants led to the isolation of a variety of leaf-closing and leaf-opening substances. And, we found that the circadian rhythmic leaf-movement of these plants is controlled by a biological clock that regulates the balance of concentration between leaf-opening and -closing substances.
\end{abstract}

Keywords: nyctinasty, legumes, biological clock, leaf-closing substance, leaf-opening substance

\section{Introduction}

In contrast with animals, plants are rooted and unable to move from one place to another by themselves. However, they are not static but sensitively respond to a variety of environmental factors such as light, temperature, and humidity, as well as to chemical substances represented by allelopathic compounds that result in visually detectable movement.

This review focuses on a variety of bioactive compounds related to the leaf movement of nyctinastic plants, whose leaves close at night and open in the daytime according to a circadian rhythm. This rhythm is regulated by a biological clock with a cycle of about 24 hours [1]. This movement is called nyctinasty. Nyctinasty is usually observed in most leguminous plants. In any event, such an exciting biological phenomenon has attracted much attention since the fourth century B.C. 
Historically, the discovery of a biological clock was based on the observation of nyctinastic movement in Mimosa pudica L. In the 18th century, a French scientist discovered that the rhythm involved in nyctinastic leaf movement was maintained even under continuous darkness in a cave [2]. He hypothesized that the leaf movement is controlled by the intrinsic rhythm of the plant. In 1880, Charles Darwin, well known for his biologically important book entitled "Origin of Species" also published an invaluable book entitled "The Power of Movement in Plants" based on experiments conducted by himself and his son Francis, using more than three hundred different kinds of plants including nyctinastic ones represented by $M$. pudica [3].

\section{Leaf-Movement Factors in Nyctinastic Plants}

Nyctinastic movement has been believed to be controlled by Schildknecht's turgorins which induce leaf-closing movement of the plants [4]. Schildknecht said that all leaf-movements are controlled by turgorin, a new class of phytohormone which regulates the turgor of the plants. However, we revealed that turgorin is not a genuine leaf movement factor. We isolated leaf-closing and -opening substance from several nyctinastic plants. Nyctinastic movement is regulated by a chemical substance that differs depending on the plant. Our present results are in accordance with Umrath et al.'s physiologically significant opinion that every family or subfamily of plants has its own leaf movement factor that is effective only for plants belonging to its own family, although they have not identified any leaf movement factor [5]. Also, we revealed that there exist leaf-opening substances together with leaf-closing ones in every nyctinastic plant. Leaf-opening substances differed in certain aspects from indole-3acetic acid (IAA) that has been believed to induce the leaf-opening of nyctinastic plants [6]: 1) the bioactivities of the leaf-opening substances $\left(c a .1 \times 10^{-6} \mathrm{M}\right)$ were much stronger than that of IAA $(>1 \times$ $10^{-4} \mathrm{M}$ ), and 2) the bioactivity of the leaf-opening substances were specific to the genus of the plant while that of IAA was nonspecific.

The presence of leaf-opening substances indicates that nyctinastic movement is controlled not only by the change in the concentration of the leaf-closing factor, but also by the competitive interaction between leaf-closing and leaf-opening substances. The idea of the co-existence of a leaf-opening substance with a leaf-closing one is very reasonable in terms of the previously mentioned result that $\mathrm{K}^{+}$ ions enter and leave plant cells via ion channels which are regulated differently [7].

\section{Summary of the Isolation of Leaf-Movement Factors}

So far, several bioactive substances controlling the leaf movement of nyctinastic plants have been identified (Figure 1) [8-17]. We have now identified five sets of leaf-closing and -opening substances from five nyctinastic plants. 


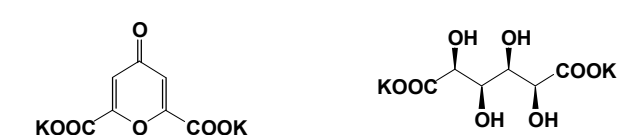

Potassium chelidonate (1) (Cassia mimosoides L., Cassia occidentalis L.)

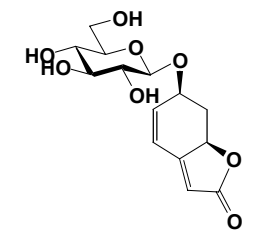

Phyllanthurinolactone (7) (Phyllanthus urinaria L.)

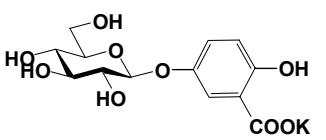

Potassium 5-O- $\beta$-glucopyranosylgentisate (9) (Mimosa pudica L.)

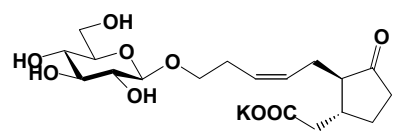

Potassium $\beta$-D-glucopyranosyl 12-hydroxy jasmonate (11) (Albizzia julibrissin Durazz)

Leaf-opening Substances

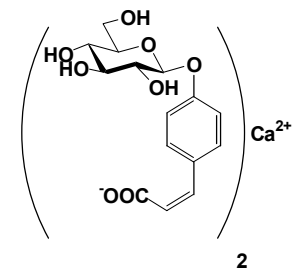

Calcium 4-O- $\beta$-D-glucopyranosyl cis-p-coumarate (4)
(Cassia mimosoides L.)

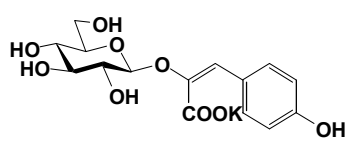

Potassium lespedezate (6) (Lespedeza cuneata G. Don)

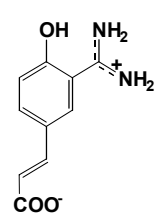

Phyllurine (3) (Phyllanthus urinaria L.)

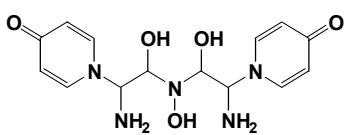

Mimopudine (10) (Mimosa pudica L.)

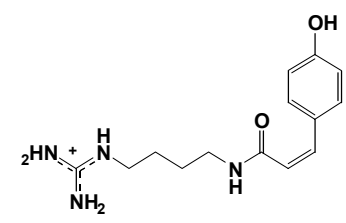

cis-p-coumaroylagmatine (12) (Albizzia julibrissin Durazz)

Figure 1. Leaf-movement factors from nyctinastic plants.

It was believed that nyctinastic movement is controlled by turgorin, a leaf-closing phytohormone common to all nyctinastic plants. However, the leaf-closing substances of nyctinastic plants were proven to be different from each other. There also exist leaf-opening substances that compete with leaf-closing ones in controlling the leaf movement of nyctinastic plants. It is important that both leafclosing and -opening substances have been isolated from the same plant, as shown in Figure 1. Therefore, each nyctinastic plant has its own leaf-opening and leaf-closing substances, and nyctinastic movement is controlled by these compounds.

These leaf-movement factors have five properties in common;

1) At the concentration of approximately $10^{-6}-10^{-7} \mathrm{M}$, all bioactive substances exert their effects only on the plants where they originate. This concentration is almost the same as that of known phytohormones, such as IAA and gibberellin.

2) The bioactivity of all leaf-movement factors is specific to the original plant from which it was isolated.

3) All leaf-closing substances have a common physiological property in that they compete with IAA at $10^{-6} \mathrm{M}$. However, much more IAA $\left(c a .10^{-4} \mathrm{M}\right)$ is required to achieve a competitive state than the natural abundance of IAA in a plant body.

4) The bioactivity of these compounds is dependent on their stereochemistry. Optically active 5 and 7 were the only bioactive ones among their stereoisomers. This suggests that a specific receptor participates in the process of signal transduction by these substances. 
5) The leaf-opening substance competes with the leaf-closing substance. When the concentration of the leaf-closing substance was higher than that of the leaf-opening substance, the leaves were closed during the day and vice versa.

\section{Chemical Control of Leaf Movement in Nyctinastic Plants}

From the viewpoint of chemical studies, the next important problem to be solved regarding nyctinasty is how these compounds control the nyctinastic leaf-movement.

We have isolated two leaf-movement factors of contrasting bioactivities from the same nyctinastic plants. The discovery of leaf-opening substances from nyctinastic plants prompted us to assume that nyctinastic leaf-movement is controlled by a competitive interaction between leaf-closing and leafopening substances. As described above, five sets of leaf-movement factors were isolated from five plants (figure 1). These bioactive substances inhibited each other competitively. Then, we assumed that the concentration of these two substances changes through a day.

Potassium D-idarate (5), a leaf-closing substance of $L$. cuneata, interacts competitively with potassium lespedezate (6), a leaf-opening substance of the same plant. When the concentration of $\mathbf{5}$ was higher than that of $\mathbf{6}$, the leaves were closed during the day, and vice versa. Furthermore, we have made an important discovery that plant extracts of all the nyctinastic plants collected during the day and at night exhibited opposite bioactivity to each other; the extract collected in the daytime exhibited leaf-opening activity, and kept the leaves open even at night, while the extract collected at night exhibited weak leaf-closing activity, and kept the leaves closed even during the daytime. It is important that the bioactivity of the plant extract completely reflected the status of the collected leaves; the plant extract collected when the leaves were closed showed leaf-closing activity and vice versa.

Presumably, these bioactive substances are not stored in the plant body, but are metabolized in the course of time. The enzymatic transformation during metabolism should be controlled by a biological clock. Thus, chemical studies on this transformation should give us an important clue to revealing the chemical control of leaf-movement by a biological clock.

We demonstrate the chemical mechanism for nyctinasty of L. cuneata G. Don, as an example [18, 19]. The extracts of L. cuneata collected during the day (around 10:00 AM) and at night (around 7:00 PM) showed inverse bioactivity; in other words, the former showed leaf-opening activity but the latter showed leaf-closing activity. These results suggest that the balance of concentration between 5 and 6 (or its cis isomer) is reversed in these two extracts. HPLC analysis revealed that the extract collected during the day contained twice as much 6 as the extract collected at night, 10:00 PM.

This two-fold increase in the concentrations of $\mathbf{6}$ is sufficient to inverse the bioactivity of the extract from our previous research on the competition experiment between $\mathbf{5}$ and $\mathbf{6}$. Thus, $\mathbf{6}$ is metabolized in the evening, and biosynthesized in the morning. Moreover, we discovered that the concentrations of 6 in the plant are inversely proportional to that of potassium 4-hydroxyphenylpyruvate (13). 
The effectiveness of the pyruvate $(13)\left(5 \times 10^{-5} \mathrm{M}\right)$ was one-hundredth of that of $6\left(8 \times 10^{-7} \mathrm{M}\right)$ inducing leaf-opening movement. The extract collected at night contained fivefold as much $\mathbf{1 3}$ as the extract collected during the day. This result strongly suggests that $\mathbf{6}$ is biosynthesized from $\mathbf{1 3}$ in the morning, and is metabolized (and deactivated) to 13 by enzymatic hydrolysis in the evening, 5:00 PM (Figure 2).

This new model of the regulation of leaf-movement was strongly supported by the measurement of $\beta$-glucosidase activities of the crude enzyme prepared from plants collected during the day and the evening. The crude enzyme was prepared according to the method of Watanabe et al [20]. We collected the leaves of L. cuneata and prepared acetone powder. We used $\mathbf{6}$ as substrate of the enzyme, and quantitatively analyzed $\mathbf{1 3}$ produced by treatment of the acetone powders by HPLC. $\beta$ - Glucosidase activity was observed only in the acetone powder prepared from the plant collected in the evening.

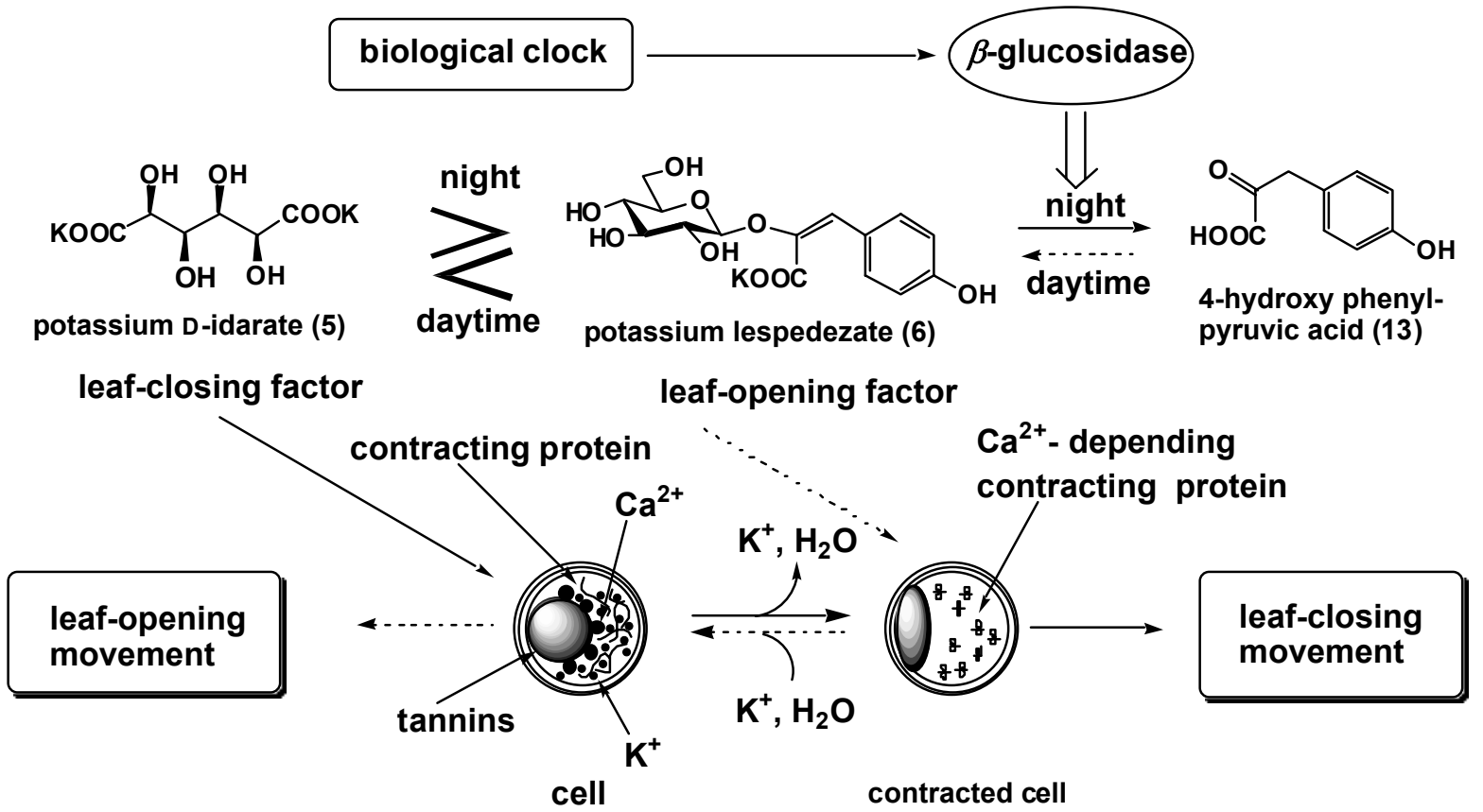

Figure 2. Chemical control of nyctinasty in Lespedeza cuneata G. Don

We propose a new model for the regulation of nyctinastic movement in L. cuneata: enzymatic transformation of leaf-movement factors (6) inversed the balance of concentration between $\mathbf{5}$ and $\mathbf{6}$, wherein a biological clock should control leaf-movement by activation or expression of the enzyme concerned in this step (Figure 2). Our study on the nyctinastic movement of L. cuneata revealed that nyctinastic leaf-movement is controlled by a biological clock through the regulation of the activity of $\beta$-glucosidase which hydrolyzes the leaf-opening substance of this plant.

A similar model would be applicable in the cases of P. urinaria [21]. In the case of P.urinaria, the leaf-closing substance, which is a glucoside, decrease in the daytime. On the other hand, the concen- 
tration of leaf-opening substance is constant during the daytime. Thus, glycoside-type leaf-closing substance would be hydrolyzed by the $\beta$-glucosidase whose activity is regulated by a biological clock.

There is some possibility that the regulation of all nyctinastic leaf-movements can be explained by only one mechanism, namely, that either the leaf-closing or -opening substance is a glucoside in all nyctinastic plants. The biological clock regulates the activity of $\beta$-glucosidase which deactivates the glucoside-type leaf-movement factor to control the internal balance of concentration between leafclosing and-opening substances.

\section{Direct Observation of the Target Cell for the Leaf-Movement Factor by Using Synthetic}

\section{Probe Compounds}

Bioactive substances controlling nyctinasty can be used for probe compounds which would be highly useful for the purification of their receptors to enable bioorganic studies of nyctinasty.

Investigation of the site where bioactive substances are perceived at the cellular level is an essential step towards the isolation and identification of the receptor molecule. A fluorescence-labeled bioactive substance is most suitable for the study of this problem, because the fluorescence-labeled compounds are widely used for the identification of receptor molecules. Then, we tried to prepare the chemical synthesis of fluorescence-labeled leaf-movement factors designed on the leaf-opening substance for the leaves of Cassia mimosoides L. The most important problem on the design of fluorescence-labeled leaf-movement factors is the unstability of $\mathbf{6}$ in the plant body, which are easily hydrolyzed by $\beta$-glucosidase. Thus, we designed a probe compound based on the structure of an artificial leaf-opening substance, potassium galactolespedezate (14) and potassium galactoisolespedezate (15), which could not be hydrolyzed in a plant body [22]. Moreover, the bioactivity of $\mathbf{1 4}$ and $\mathbf{1 5}$ was as strong as the one of natural product.

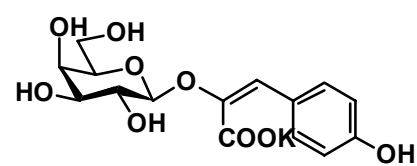

Potassium galactolespedezate $\left(14,1 \times 10^{-6} \mathrm{M}\right)$

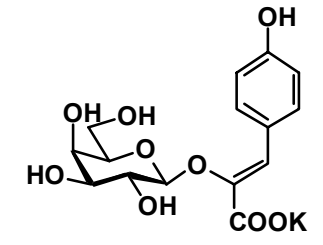

Potassium galactoisolespedezate $\left(15,1 \times 10^{-6} \mathrm{M}\right)$

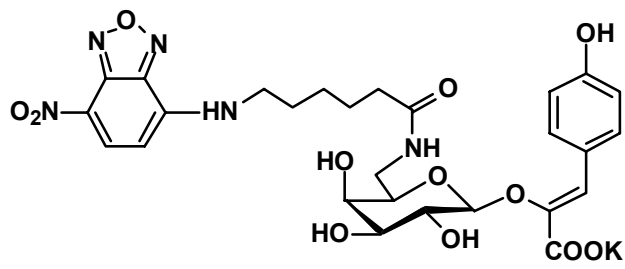

$17\left(5 \times 10^{-6} \mathrm{M}\right)$

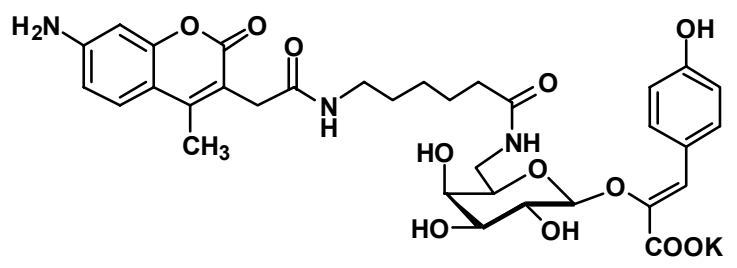

$16\left(1 \times 10^{-5} \mathrm{M}\right)$

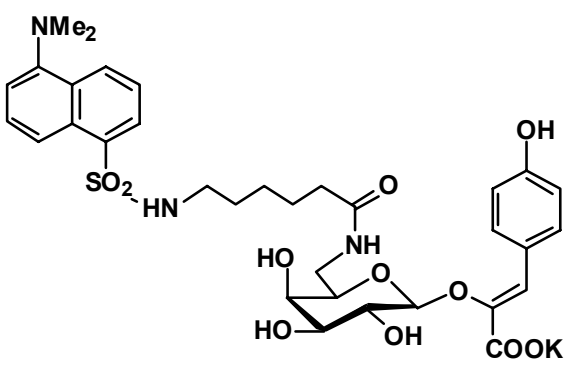

$18\left(5 \times 10^{-6} \mathrm{M}\right)$ 
The introduction of a large fluorescent dye to artificial leaf-opening substances requires careful consideration of the structure-activity relationship in $\mathbf{6}$. From the careful reseach on the structure-activity relationship, it is expected that the introduction of a large fluorescent functional group in the hydroxyl group at the 6' position of the galactose moiety would not weaken the bioactivity of $\mathbf{1 4}$ and $\mathbf{1 5}$ to any extent. Moreover, because of the resistance to the esterase in a plant body, an amide bond would be better than an ester bond to connect a fluorescent dye with $\mathbf{1 4}$ and $\mathbf{1 5}$. Thus, to introduce a fluorescent dye at the 6' position of $\mathbf{1 4}$ (or 15), we should convert the hydroxy group on the 6' position of $\mathbf{1 4}$ (or 15) into an amino group. After many trials, we developed probe compounds with AMCA (16), NBD (17), and dansyl (18) of which bioactivity was one-fifth to one-tenth as strong as $\mathbf{1 4}$ or $\mathbf{1 5}$ [23, 24].

We used probe $\mathbf{1 6}$ for the detection of the target cell for the leaf-movement factor [24]. On the other hand, probe $\mathbf{1 7}$ and $\mathbf{1 8}$, the fluorescence of which is similar to the background one (autofluorescence) of the plant tissue under a fluorescence microscope, was inappropriate for the fluorescence study. A leaf of $C$. mimosoides was cut by a microslicer perpendicular to the petiole to a thickness of thirty micrometers. Then the section was incubated with fluorescent probes. Then, the stained section was monitored by using a fluorescence microscope with an appropriate filter. Figure 3 shows photographs of the plant sections under a fluorescence microscope. Strong autofluorescence was observed in the plant section. However, when the section was treated with probe compound (16), the staining pattern for 16 was observed only in the motor cells contained in pulvini (Figure 3). No other part of the plant section showed the fluorescent stain for 16. Also, no stain was observed in the control section, which was treated with a solution containing no $\mathbf{1 6}$. The fluorescence of $\mathbf{1 6}$ was observed on the surface of the motor cells.
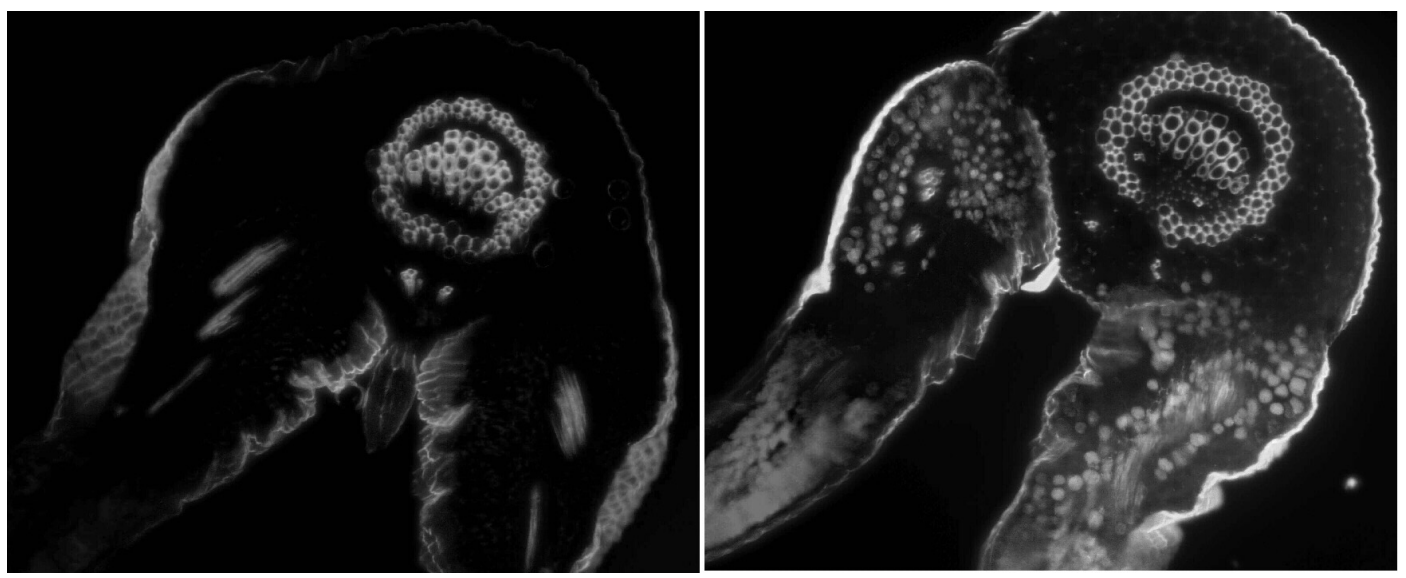

Figure 3. Binding experiment using a fluorescentprobe compound

Motor cells in the pulvini of the plant exert their function by swelling and shrinking in the process of leaf-opening and -closing and play a central role in the plant leaf-movement. ${ }^{23}$ The ion fluxes followed by massive water fluxes across the plasma membrane of these cells produce the swelling and 
shrinking behavior of the motor cells. Our present results suggest that the binding site for $\mathbf{1 6}$ should exist on the plasma membrane of the motor cell.

\section{Conclusion}

Leaf-movement in nyctinastic plants has long been believed to be controlled by a common phytohormone. However, our result contradicts this theory. Indeed, we advance a new theory of the chemical control of nyctinastic leaf movement; nyctinastic leaf-movement is controlled by the balance of concentration between two bioactive substances, leaf-opening and -closing substances, which is inversed through the day according to the rhythm created by their biological clock. A biological clock regulates this balance through the control of the $\beta$-glucosidase activity (Figure 5) [22].
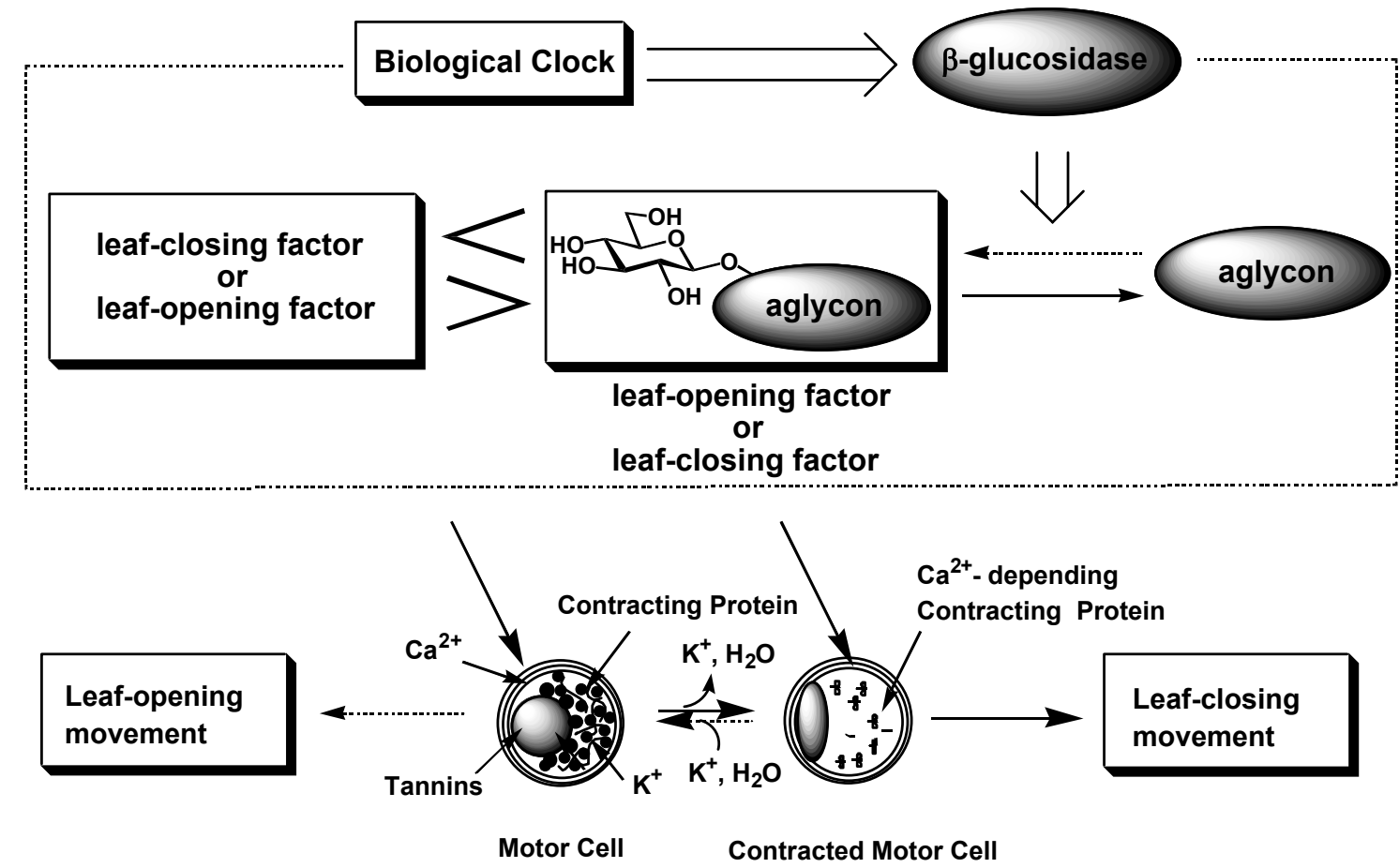

Figure 5. Universal mechanism of nyctinasty controlled by a biological clock.

Our leaf-movement factors, whose concentrations have been proven to change according to a circadian rhythm, should be genuine chemical signals that control leaf-movement in nyctinastic plants from a physiological viewpoint. Moreover, we have shown that leaf-movement factor directly interact with motor cell by using fluorescence-labeled probe compound.So far, the behavior of our leafmovement factor can fully account for this physiological phenomenon in the molecular level. The next issue of our research is focused on investigation of the role of leaf-movement factors in this biological event, especially, the signal transduction after the perception of the eaf-movement factor by the receptor of the leaf-movement factor. Our present result will develop the science of this field from plant physiology into bioorganic chemistry. And we expect that these results would bring us a clue in the investigation of the nature of the biological clock in highly organized plants. 


\section{Acknowledgments}

We are indebted to the Ministry of Education, Science, Sports and Culture (Japan) for Grant-in-Aid for Scientific Research (No. 12045259 and No. 12680598), Pioneering Research Project in Biotechnology given by the Ministry of Agriculture, Forestry and Fisheries, Mishima-Kaiun Foundation, and Inamori Foundation for financial support.

\section{References}

1. Du Fay, Observation botanique. Histoire de L'Academie Royale des Sciences (Paris 1729), p.35.

2. Bünning, E. The Physiological Clock, Third Edition; English Univ. Press: London, 1973.

3. Darwin, C. The Power of Movement in Plants; John Murray: London, 1880.

4. Schildcknecht, H. Angew. Chem., Int. Ed. Engl., 1983, 22, 695-710.

5. Bielenberg, W.; Esterbauer, H.; Hayn, M.; Umrath, K. Phyton, 1984, 24, 1-10.

6. Watanabe, S.; Umrath, K. Phyton, 1983, 23, 49-54.

7. Lee, Y. In The Pulvinus in Motor Organ for Leaf Movement; Satter, R. L.; Gorton H. L.; Vogelmann, T. C., Ed.; American Society of Plant Physiologists: Rockville, 1990, p. 130-141.

8. Miyoshi, E.; Shizuri, Y.; Yamamura, S. Chemistry Lett., 1987, 511-514.

9. Ueda, M.; Ohnuki, T.; Yamamura, S. Phytochemistry, 1998, 49, 633-635.

10. Ueda, M.; Shigemori-Suzuki, T.; Yamamura, S. Tetrahedron Lett., 1995, 36, 6267-6270.

11. Ueda, M.; Asano, M.; Yamamura, S. Tetrahedron Lett., 1998, 39, 9731-9734.

12. Ueda, M.; Ohnuki, T.; Yamamura, S. Tetrahedron Lett., 1997, 38, 2497-2500.

13. Shigemori, H.; Sakai, N.; Miyoshi, E.; Shizuri, Y.; Yamamura, S. Tetrahedron Lett., 1989, 30, 3991-3994.

14. Ueda, M.; Tashiro, C.; Yamamura, S. Tetrahedron Lett., 1997, 38, 3253-3256.

15. Ueda, M.; Yamamura, S. Tetrahedron Lett., 1999, 40, 7823-7826.

16. Ueda, M.; Yamamura, S. Tetrahedron Lett., 1999, 40, 353-356.

17. Ueda, M.; Yamamura, S. Tetrahedron Lett., 1999, 40, 2981-2984.

18. Ohnuki, T.; Ueda, M.; Yamamura, S. Tetrahedron, 1998, 54, 12173-12184.

19. Ueda, M.; Ohnuki, T.; Yamamura, S. Chemistry Lett., 1998, 179-180.

20. Watanabe, H.; Watanabe, S.; Nakajima, R.; Moon, J.-H.; Shimokihara, K.; Inagaki, J.; Etoh, H.; Asai, T. Biosci. Biotech. Biochem., 1993, 57, 1101-1106.

21. Ueda, M.; Asano, M.; Sawai, Y.; Yamamura, S. Tetrahedron, 2000, 55, 5781-5792.

22. Ueda, M.; Sawai, Y.; Yamamura, S. Tetrahedron Lett., 2000, 40, 3433-3436.

23. Ueda, M.; Sawai, Y.; Wada, Y.; Yamamura, S. Tetrahedron, 2000, 40, 5123-5130.

24. Ueda, M.; Wada, Y.; Yamamura, S. Tetrahedron Lett., 2001, 41, 3869-3872. 